\title{
A Chemical Approach to 3-D Lithographic Patterning of Si and Ge Nanocrystals
}

I.D. Sharp,,${ }^{a, b}$ Q. Xu ${ }^{a, b}$ D.O. Yi, ${ }^{a, c}$ C.Y. Liao, ${ }^{a, b}$ J.W. Ager III, ${ }^{a}$ J.W. Beeman, ${ }^{a}$ K.M. Yu, ${ }^{a}$ J.T. Robinson, ${ }^{\mathrm{a}, \mathrm{b}}$ O.D. Dubón, ${ }^{\mathrm{a}, \mathrm{b}}$ D.C. Chrzan, ${ }^{\mathrm{a}, \mathrm{b}}$ and E.E. Haller ${ }^{\mathrm{a}, \mathrm{b}}$

a) Materials Sciences Division, Lawrence Berkeley National Laboratory, Berkeley, CA 94720

${ }^{b)}$ Materials Science and Engineering Department, University of California, Berkeley, Berkeley, CA 94720

${ }^{c}$ Applied Science and Technology Group, University of California, Berkeley, Berkeley CA 94720

\begin{abstract}
Ion implantation into silica followed by thermal annealing is an established growth method for $\mathrm{Si}$ and Ge nanocrystals. We demonstrate that growth of Group IV semiconductor nanocrystals can be suppressed by co-implantation of oxygen prior to annealing. For Si nanocrystals, at low $\mathrm{Si} / \mathrm{O}$ dose ratios, oxygen co-implantation leads to a reduction of the average nanocrystal size and a blue-shift of the photoluminescence emission energy. For both Si and Ge nanocrystals, at larger $\mathrm{Si} / \mathrm{O}$ or $\mathrm{Ge} / \mathrm{O}$ dose ratios, the implanted specie is oxidized and nanocrystals do not form. This chemical deactivation was utilized to achieve patterned growth of $\mathrm{Si}$ and Ge nanocrystals. Si was implanted into a thin $\mathrm{SiO}_{2}$ film on a $\mathrm{Si}$ substrate followed by oxygen implantation through an electron beam lithographically defined stencil mask. Thermal annealing of the co-implanted structure yields two-dimensionally patterned growth of Si nanocrystals under the masked regions. We applied a previously developed process to obtain exposed nanocrystals by selective $\mathrm{HF}$ etching of the silica matrix to these patterned structures. Atomic force microscopy (AFM) of etched structures revealed that exposed nanocrystals are not laterally displaced from their original positions during the etching process. Therefore, this process provides a means of achieving patterned structures of exposed nanocrystals. The possibilities for scaling this chemical-based lithography process to smaller features and for extending it to 3-D patterning is discussed.
\end{abstract}

\section{INTRODUCTION}

The ability to precisely control nanocrystal growth sites and/or their placement is of critical importance for realization of functional nanocrystal-based devices, for single and ensemble nanocrystal measurements, and possibly for additional control of nanocrystal sizes. Patterning of solution-processed nanocrystals has been achieved by a number of techniques.[1-6] However, production of colloidal Group IV semiconductor nanocrystals is difficult and it is often preferable to form them within a solid matrix, usually $\mathrm{SiO}_{2}$. While patterned growth of embedded nanocrystals has been realized, only a limited number of techniques have been explored thus far.[7-10] Here, we demonstrate the patterned growth of ion beam synthesized $\mathrm{Si}$ and Ge nanocrystals. Chemical deactivation of the nucleating specie is achieved by coimplantation of oxygen through a lithographically defined mask. Furthermore, we show that by varying the oxygen dose, the light emission energy of Si nanocrystals may be tuned. This technique could be further utilized for compositional variation across a single sample to form, for 
example, $\mathrm{Si}_{1-\mathrm{x}} \mathrm{Ge}_{\mathrm{x}}$ alloy nanocrystals or compound semiconductor nanocrystals with spatially varied compositions.

\section{EXPERIMENTAL}

Experiments to determine the effect of various oxygen co-implantation doses on the growth of Si and Ge nanocrystals were performed prior to patterning. Unpatterned samples were formed by blanket multi-energy ion implantation of ${ }^{28} \mathrm{Si}^{+}$at $50 \mathrm{keV}$ to $2.5 \times 10^{16} \mathrm{~cm}^{-2}$ and $35 \mathrm{keV}$ to $1.5 \times 10^{16} \mathrm{~cm}^{-2}$ or ${ }^{74} \mathrm{Ge}$ at $120 \mathrm{keV}$ to $2 \times 10^{16} \mathrm{~cm}^{-2}, 80 \mathrm{keV}$ to $1.2 \times 10^{16} \mathrm{~cm}^{-2}$, and $50 \mathrm{keV}$ to $1 \times 10^{16} \mathrm{~cm}^{-2}$ into $\mathrm{SiO}_{2}$. Oxygen co-implantation was performed with doses ranging from 0 to $4 \times 10^{16} \mathrm{~cm}^{-2}$ with an accelerating voltage of $32 \mathrm{keV}$ for all implants. Ge (Si) implanted samples were annealed at $900{ }^{\circ} \mathrm{C}$ for $1 \mathrm{~h}\left(1100^{\circ} \mathrm{C}\right.$ for $\left.30 \mathrm{~min}\right)$ under Ar and were quenched under cold running water. Structural properties of Ge nanocrystals were probed using Raman spectroscopy and the optical properties of Si nanocrystals were determined using photoluminescence (PL). We note that interference from the Si substrate precludes Raman spectroscopic characterization of the Si nanocrystals and the lack of Ge nanocrystal-related PL emission precludes optical emission characterization of Ge nanocrystals.

Patterned nanocrystal growth was first achieved using ink pen markings on the surface of a Ge implanted sample. This preliminary experiment was performed to verify the principle of nanocrystal patterning using oxygen co-implantation. A blanket Ge implant into a thin $\mathrm{SiO}_{2}$ film on Si was performed using the conditions described in the previous paragraph. An ink pen was used to "lithographically" pattern the sample, and oxygen was implanted through the "mask" at $32 \mathrm{keV}$ to $4 \times 10^{16} \mathrm{~cm}^{-2}$. After verification of patterned nanocrystal growth using this technique, additional experiments were performed using a more advanced mask.

Sub-micron patterned nanocrystal growth was achieved using an electron beam lithographically (EBL) defined stencil mask. Low-stress silicon nitride windows, $100 \mathrm{~nm}$ thick, on a Si substrate were patterned with arrays of $200 \mathrm{~nm}$ square holes spaced $900 \mathrm{~nm}$ apart by EBL and reactive ion etching. Though patterned growth could, in principle, be achieved by direct implantation of Si through the stencil mask, co-implantation of oxygen, a lighter element, was chosen for this study in order to minimize damage to the mask. ${ }^{28} \mathrm{Si}^{+}$was first implanted with the doses and energies given above into a $500 \mathrm{~nm}$ thick $\mathrm{SiO}_{2}$ thin film on a $\mathrm{Si}$ substrate without masking the surface. The stencil mask was then placed in direct contact with the surface of the $\mathrm{Si}$ implanted sample and ${ }^{16} \mathrm{O}^{+}$was implanted through the mask at $32 \mathrm{keV}$ to $4 \times 10^{16} \mathrm{~cm}^{-2}$. The sample was then annealed at $1200{ }^{\circ} \mathrm{C}$ for $1 \mathrm{~h}$ under Ar.

To verify patterned nanocrystal formation, samples were first etched in a solution of $1: 1$ $49 \% \mathrm{HF}: \mathrm{H}_{2} \mathrm{O}$. As described elsewhere, the etching process selectively removes the $\mathrm{SiO}_{2}$ film while preserving the nanocrystals on the underlying Si substrate.[11] Height data from the etched samples were obtained using atomic force microscopy (AFM).

\section{RESULTS AND DISCUSSION}

Figure 1(a) shows Raman spectra of ${ }^{74} \mathrm{Ge}$ nanocrystals embedded in $\mathrm{SiO}_{2}$ after $\mathrm{O}$ coimplantation to $0,2.1$, and $4.2 \times 10^{16} \mathrm{~cm}^{-2}$. With no oxygen co-implantation the Raman spectrum is asymmetrically broadened due to phonon confinement. With the addition of $2.1 \times 10^{16} \mathrm{~cm}^{-2} \mathrm{O}$, the spectrum FWHM increases from $11 \mathrm{~cm}^{-1}$ to $20 \mathrm{~cm}^{-1}$, indicating a reduction of the average 

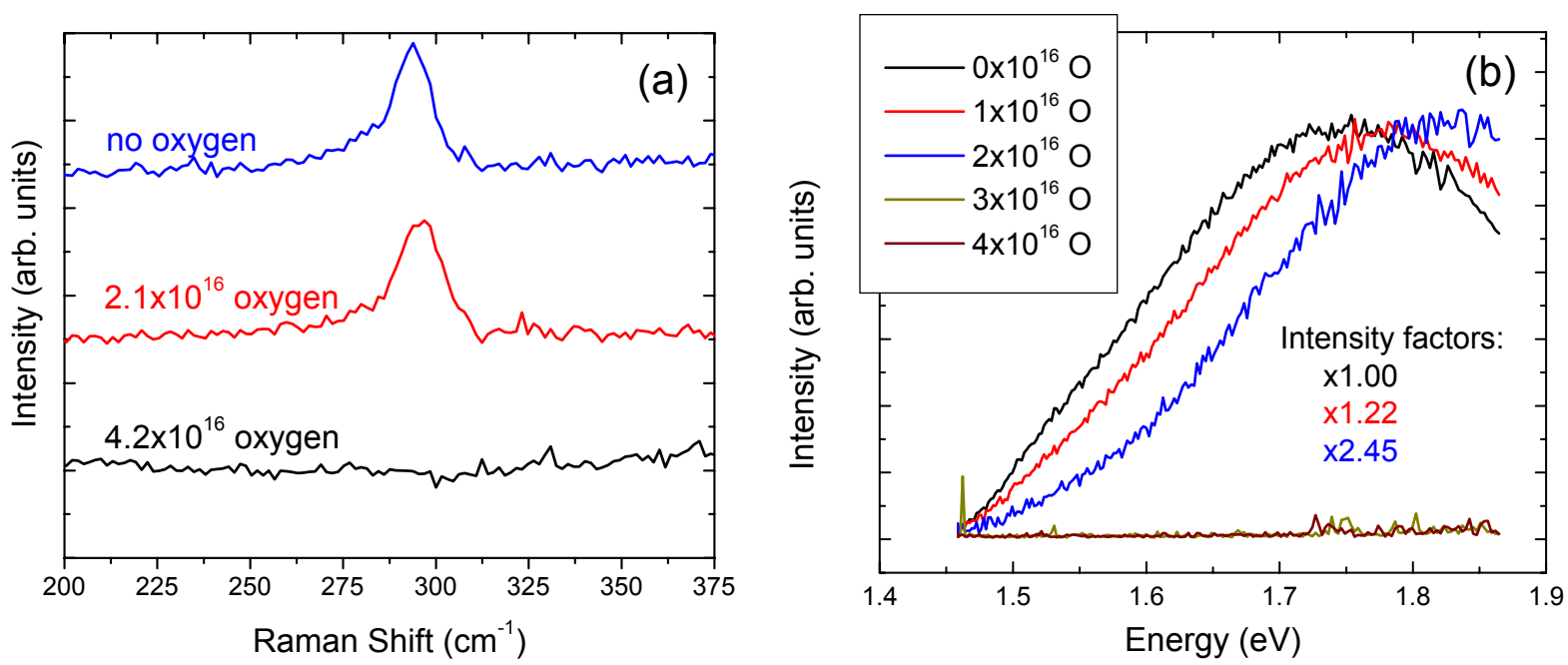

Figure 1: (a) Raman spectra from samples implanted with a total dose of $4.2 \times 10^{16} \mathrm{~cm}^{-2} \mathrm{Ge}$, coimplanted with various $\mathrm{O}$ doses, and annealed at $900{ }^{\circ} \mathrm{C}$ for $1 \mathrm{~h}$. The FWHM of the Ge nanocrystal Raman peak increases with the addition of $\mathrm{O}$, indicating a decrease of the average crystal size. No nanocrystals form with O:Ge dose ratios at and exceeding 1:1. (b) Photoluminescence spectra from samples implanted with a total dose of $4 \times 10^{16} \mathrm{~cm}^{-2} \mathrm{Si}$, coimplanted with a range of $\mathrm{O}$ doses, and annealed at $1100{ }^{\circ} \mathrm{C}$ for $30 \mathrm{~min}$. The emission energy increases with increasing $\mathrm{O}$ dose, suggesting a decrease of the average crystal size. The peak intensity decreases with increasing $\mathrm{O}$ dose (see "Intensity factors"), until luminescence disappears at an $\mathrm{O}: \mathrm{Si}$ dose ratio of 3:4.

nanocrystal size. At $\mathrm{O}: \mathrm{Ge}$ dose ratios of and exceeding 1:1, the implanted Ge is chemically deactivated by oxygen, and no nanocrystals form. The detailed dependence of the Ge nanocrystal size distribution on $\mathrm{O}$ co-implant dose is under investigation.

Photoluminescence measurements from Si nanocrystals indicate a similar dependence of the average nanocrystal size on the $\mathrm{O}$ dose. Figure 1(b) shows PL spectra obtained for a range of $\mathrm{O}: \mathrm{Si}$ dose ratios after annealing at $1100{ }^{\circ} \mathrm{C}$ for $30 \mathrm{~min}$. As the $\mathrm{O}$ dose increases, the $\mathrm{PL}$ emission energy increases. However, at and above $3 \times 10^{16} \mathrm{~cm}^{-3} \mathrm{O}$, nanocrystals no longer form and no luminescence is observed. It should be noted that the luminescence energy shift is quite small $(\sim 100 \mathrm{meV})$. It is likely that for the nanocrystal size range investigated here (average diameter $>$ $3 \mathrm{~nm}$ ), luminescence originates from size-dependent oxygen-related defect centers at the nanocrystal/matrix interface rather than from confined excitons within the nanocrystal.[12] Further studies are underway on larger nanocrystals, which can be obtained by annealing at higher temperatures, to determine the effect of oxygen dose on confinement-based luminescence.

Based on the variable dose $\mathrm{O}$ co-implant experiments, a dose ratio of 1:1 O:Ge was selected for patterned chemical deactivation of Ge. Figure 2 shows an AFM image of patterned Ge nanocrystals on a Si substrate. A step feature was "lithographically" defined using an ink pen. After O co-implantation, annealing, and HF etching, the nanocrystal pattern is retained on the underlying Si substrate with nanocrystals in the masked region and none in the O coimplanted region. Importantly, the interface between the two regions is sharp. This demonstrates that the nanocrystals are not swept over the surface during the etching procedure. 


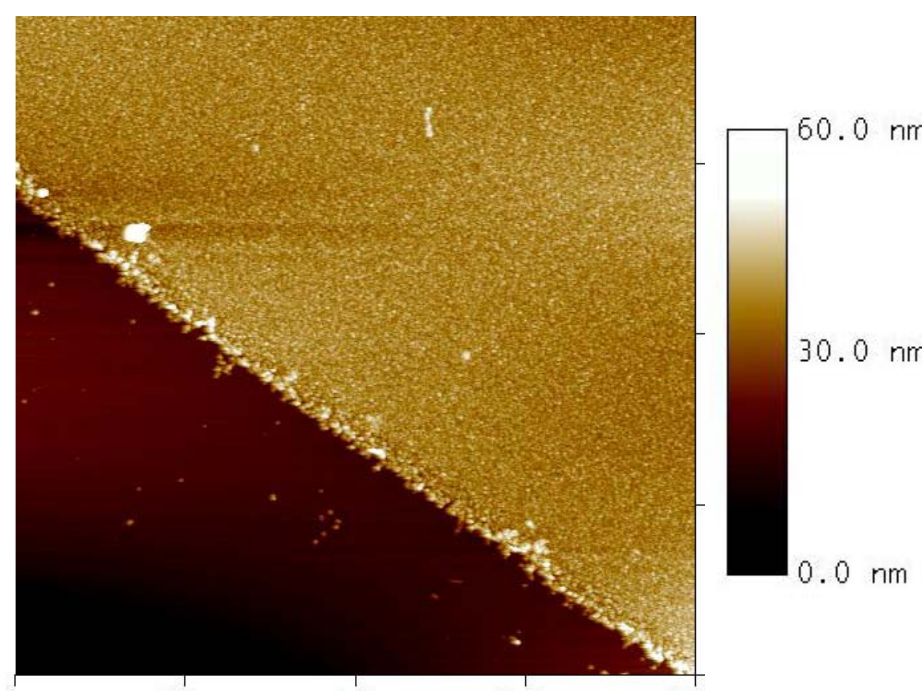

Figure 2: A $10 \times 10 \mu \mathrm{m}$ AFM scan demonstrating patterned $\mathrm{Ge}$ nanocrystal formation after selective etching of the film to obtain freestanding nanocrystals. The bottom left of the image shows the $\mathrm{Si}$ substrate and corresponds to the unmasked region with $\mathrm{Ge}$ and $\mathrm{O}$ coimplantation. The upper right portion of the images shows nanocrystals sitting on a substrate, and corresponds to the masked region with Ge only implantation. A sharp interface between the masked and unmasked region is visible.

Following verification of successful patterning and development of an appropriate characterization technique, the more advanced EBL-defined stencil mask was used for patterned growth of Si nanocrystals. Again, a dose ratio of 1:1 O:Si was selected for implantation through the mask. Figure 3 shows an AFM image of the nanocrystals adhering to the Si substrate after O co-implantation through regularly-spaced $200 \mathrm{~nm}$ square holes, annealing, and HF etching. Suppression of nanocrystal formation in the $\mathrm{O}$ co-implanted regions is apparent and the pattern is retained remarkably well even after the etching process. This result demonstrates that the process presented here is well-suited for patterned nanocrystal growth.

The scalability of this process is of significant importance and is straightforward to evaluate theoretically.[13] Lateral straggle of the $\mathrm{O}$ atoms upon implantation through the mask will ultimately limit the achievable feature size. To quantitatively analyze this effect, Gaussian lateral straggling is assumed and the normalized lateral distribution at the projected range is calculated using parameters obtained from SRIM.[14] The concentration distribution is normalized to the concentration at the projected range with no masking. Figure 4(a) shows a plot of the normalized peak concentration at the projected range under the center of a square mask window for a $32 \mathrm{keV} \mathrm{O}$ implantation. For large mask sizes, the normalized peak concentration is not affected by the presence of the mask because straggling effects are limited to regions near the

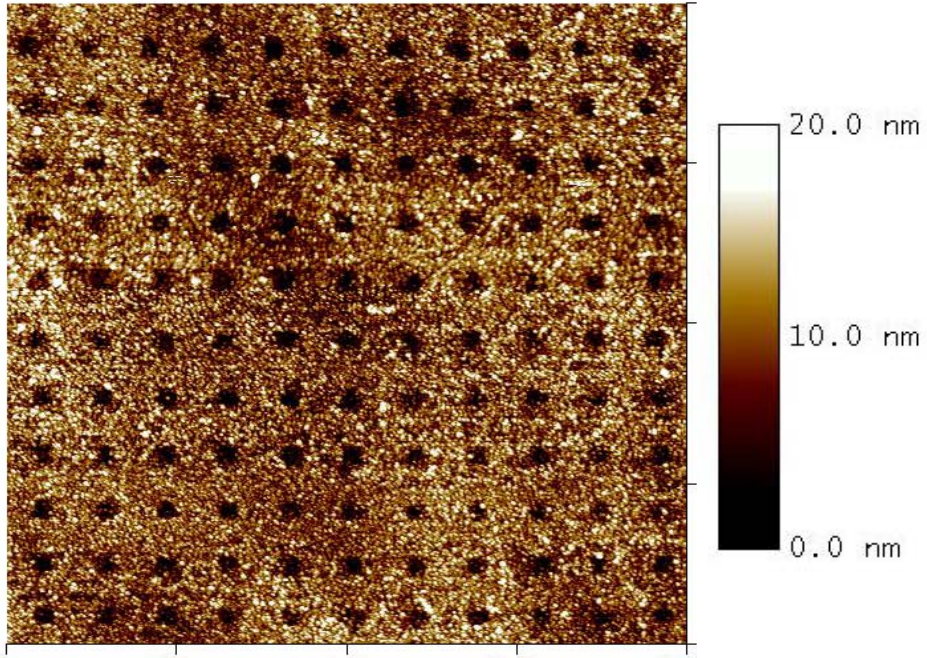

Figure 3: A $10 \times 10 \mu \mathrm{m}$ AFM scan showing patterned growth of $\mathrm{Si}$ nanocrystals after selective etching of the oxide matrix. Oxygen was coimplanted through a stencil mask with $200 \mathrm{~nm}$ square holes spaced 900 $\mathrm{nm}$ apart. The co-implanted pattern is absent of nanocrystals, as expected. This verifies that the process of chemical deactivation of $\mathrm{Si}$ and $\mathrm{Ge}$ for patterned nanocrystal growth is scalable to small feature sizes. 

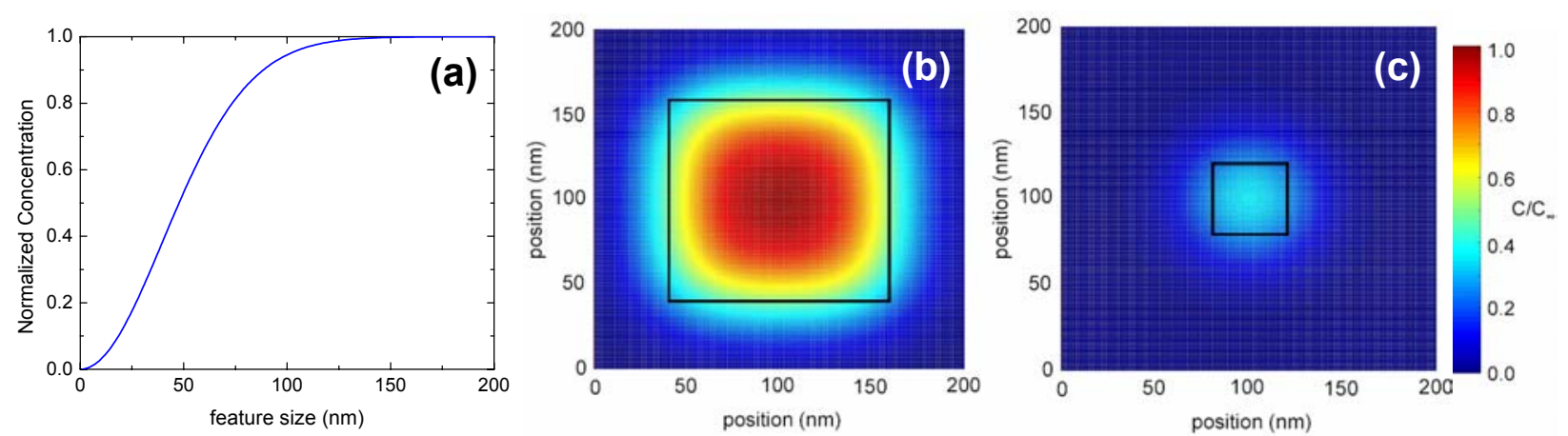

Figure 4: (a) Calculated oxygen peak concentration under the center of a square mask at the projected range normalized to the concentration with no mask present for $32 \mathrm{keV}$ implantation. At feature sizes smaller than $\sim 100 \mathrm{~nm}$, the achieved concentration drops rapidly due to lateral ion straggling. (b) Calculated normalized concentration map for $\mathrm{O}$ implantation through a $120 \mathrm{~nm}$ square mask. (c) Calculated normalized concentration map for $\mathrm{O}$ implantation through a $40 \mathrm{~nm}$ square mask.

edge of the mask. However, as mask dimensions become comparable to the lateral straggling length, much of the implanted $\mathrm{O}$ is lost under the mask. Figures 4(b) and 4(c) show the normalized concentration distributions at the projected range for $120 \mathrm{~nm}$ and $40 \mathrm{~nm}$ mask sizes, respectively. From these implant simulations, it is apparent that patterning using masks with 120 $\mathrm{nm}$ features is likely possible, whereas $40 \mathrm{~nm}$ features are not. It should be noted, however, that even for masks with features sizes above $100 \mathrm{~nm}$, the resulting pattern in the sample could be strongly affected by straggling. These simulations were performed assuming $32 \mathrm{keV} \mathrm{O}$ implantation. However, lower energy implants yield smaller straggling and some improvement of the scalability should be possible by reducing the implant energy. In addition, implantation of heavier species, such as direct $\mathrm{Si}$ or Ge implantation through a mask, would allow for much smaller features since the straggle is significantly smaller for heavier species. Unfortunately, due to greater surface sputtering from heavier ion implants, the mask requirements become more stringent.

The results presented above demonstrate 2-D patterning of nanocrystal systems. However, control over the third dimension, depth, is also desirable. This may be achieved via implantation into silicon-on-insulator (SOI) structures. The implanted specie, either $\mathrm{Si}$ or $\mathrm{Ge}$, would be fully soluble in the Si substrate and the Si surface layer. However, nanocrystals could grow in the buried oxide layer. The thickness of this oxide and the surface Si layer, in conjunction with masked implantation, would provide a means of controlling nanocrystal placement in all three dimensions. Furthermore, the implanted concentration within the buried oxide would not have a significant depth dependence since the tails of the implanted concentration distribution could be confined within the Si layers. Results of experiments to achieve 3D ordered growth will be presented in-depth elsewhere.

\section{CONCLUSION}

Patterned chemical deactivation of $\mathrm{Si}$ and $\mathrm{Ge}$ by $\mathrm{O}$ co-implantation was utilized to achieve ordered growth of Group IV semiconductor nanocrystals. Furthermore, low dose O coimplants can be used to tune nanocrystal sizes, and thus their size-dependent properties. 
An electron beam lithographically defined stencil mask was used to show that the coimplantation process can be applied to very small features. Theoretical modeling suggests that this process is suitable for features at and above approximately $100 \mathrm{~nm}$ in size, but lateral straggling of $\mathrm{O}$ atoms precludes fabrication of smaller features. However, much smaller features should be possible by direct implantation of Si or Ge through a mask because the lateral straggling is smaller for heavier species. Experiments are underway to form ordered 3D features by integration of patterned implantation and SOI technology.

\section{ACKNOWLEDGEMENTS}

This work was supported in part by US NSF Grant Nos. DMR-0405472 and EEC-0085569, and in part by the Director, Office of Science, Office of Basic Energy Sciences, Division of Materials Sciences and Engineering, of the U.S. Department of Energy under Contract No. DE-AC02$05 \mathrm{CH} 11231$.

\section{REFERENCES}

[1] Y. Cui, M. T. Bjork, J. A. Liddle, C. Soennichsen, B. Boussert and A. P. Alivisatos, Nano Lett. 4, 1093 (2004).

[2] M. A. Islam and I. P. Herman, Appl. Phys. Lett. 80, 3823 (2002).

[3] N. Lu, X. Chen, D. Molenda, A. Naber, H. Fuchs, D. V. Talapin, H. Weller, J. Mueller, J. M. Lupton, J. Feldmann, A. L. Rogach and L. Chi, Nano Lett. 4, 885 (2004).

[4] H. O. Jacobs and G. M. Whitesides, Science 291, 1763 (2001).

[5] J. Liu, T. Lee, D. B. Janes, B. L. Walsh, M. R. Melloch, J. M. Woodall, R. Reifenberger and R. P. Andres, Appl. Phys. Lett. 77, 373 (2000).

[6] K. Y. Suh, A. Khademhosseini, G. Eng and R. langer, Langmuir 20, 6080 (2004).

[7] A. Meldrum, K. S. Buchanan, A. Hryciw and W. White, Adv. Mater. 16, 31 (2004).

[8] K. Sun, S. Zhu, R. Fromknecht, G. Linker and L. M. Wang, Mater. Lett. 58, 547 (2004).

[9] I. Berbezier, A. Karmous, A. Ronda, T. Stoica, L. Vescan, R. Geurt, A. Olzierski, E. Tsoi and A. G. Nassiopoulou, J. Phys.: Conf. Ser. 10, 73 (2005).

[10] J. Valenta, R. Juhasz and J. Linnros, Appl. Phys. Lett. 80, 1070 (2002).

[11] I. D. Sharp, Q. Xu, C. Y. Liao, D. O. Yi, J. W. Beeman, Z. Liliental-Weber, K. M. Yu, D. N. Zakharov, J. W. A. III, D. C. Chrzan and E. E. Haller, J. Appl. Phys. 97, 124316 (2005).

[12] J. S. Biteen, N. S. Lewis, H. A. Atwater and A. Polman, Appl. Phys. Lett. 84, 5389 (2004).

[13] S. Furukawa, H. Matsumura and H. Ishiwara, Jpn. J. Appl. Phys. 11, 134 (1972).

[14] J. F. Ziegler, J. P. Biersack and W. Littmark, The Stopping Range of Ions in Matter. (Pergamon Press, New York, 1985). 